\title{
Sustentabilidade no Ambiente de Startups: Revisão Sistemática de Publicações Internacionais
}

\section{Sustainability in Startups: Systematic Review of International Publications}

RENATA MENDES DE OLIVEIRA D

ILSE MARIA BEUREN

\section{RESUMO}

Este estudo analisa as abordagens das pesquisas publicadas em periódicos internacionais sobre sustentabilidade no ambiente de startups. Uma revisão sistemática foi realizada nos artigos sobre a temática publicados nos últimos vinte anos (1999-2018), disponíveis na base de dados da Scopus. Além de identificar o período com maior número de publicações, os periódicos que se sobressaíram e a autoria dos artigos, analisaram-se aspectos metodológicos das pesquisas e o objetivo de cada artigo. Os resultados revelaram que as publicações sobre a temática se tornaram mais frequentes em anos recentes, apenas dois periódicos se destacaram, e não se observou formação de redes de autores. Nos aspectos metodológicos, predominaram pesquisas desenvolvidas por meio de estudos de casos e levantamentos, com coleta de dados a partir de entrevistas, documentos e questionários. Os objetivos declarados nos artigos apontam predominância da abordagem econômica da sustentabilidade, em detrimento das abordagens social e ambiental, que juntas formam o Triple Bottom Line. Não foi observado um eixo quanto ao que é objetivado pelos artigos, o que inibe inferências quanto às tendências de pesquisas sobre sustentabilidade em startups. No entanto, isso representa uma importante contribuição, aponta uma lacuna de pesquisa relativa aos aspectos sustentáveis de startups.

Palavras-chave: Sustentabilidade; Triple Bottom Line; Startups. 


\section{Abstract}

This study analyzes the approaches of research published in international journals about sustainability in startups through a systematic review of articles on the subject, published in the last twenty years (1999-2018), available in the Scopus database. In addition to identifying the period with the largest number of publications, the outstanding journals and the authorship of the articles, the methodological aspects of the research and the purpose of each article were analyzed. The results revealed that publications on the subject have become more frequent in recent years, they are not concentrated in journals, only two journals stood out, and there isn't formation of authors networks. The methodological aspects are predominantly composed of research developed through case studies and surveys, with data collection from interviews, documents and questionnaires. The objectives stated in the articles analyzed are mainly related to the economic approach to sustainability, to the detriment of the social and environmental approaches, which together form the Triple Bottom Line. No axis was observed as to what is objectified by the articles, which restrains inferences about research trends regarding sustainability in startups. However, this represents an important contribution, identifying a research gap related to the sustainable aspects of startups.

Keywords: Sustainability; Triple Bottom Line; Startups.

\section{INTRODUÇão}

As startups podem ser consideradas como uma resposta para diferentes problemas sociais e ambientais (HALL; DANEKE; LENOX, 2010; PACHECO; DEAN; PAYNE, 2010). Essas organizações desempenham um importante papel para a transformação da sociedade ao colaborar no processo de introdução de produtos ou processos com altos benefícios ambientais ou sociais (SCHALTEGGER; WAGNER, 2011). Além disso, contribuem para o desenvolvimento de ações sustentáveis, inovação em modelos de negócios (BOCKEN, 2015).

A inovação em modelos de negócios oferece condições para a integração de elementos relacionados à sustentabilidade junto aos propósitos organizacionais (SHORT et al., 2014). O propósito conjunto desses elementos é gerar vantagem competitiva por meio 
da criação de valor superior, o que contribui positivamente para a organização e minimiza os danos ao meio ambiente e à sociedade (BOCKEN; RANA; SHORT, 2015).

Bocken et al. (2014) destacam que as pesquisas sobre sustentabilidade consideram diferentes abordagens, mas que todas acabam por contribuir de algum modo com o processo de inovação do modelo de negócios para a sustentabilidade, destacando-se questões como melhorias na ecoeficiência (produção mais limpa), criação de valor a partir de resíduos, sistemas de serviços de produtos, soluções empresariais sociais, entre outras.

As pesquisas que abordam sobre o contexto de startups investigaram diferentes aspectos, como, inovação (WEIBLEN; CHESBROUGH, 2015; DULLIUS; SCHAEFFER, 2016), sistema de estrutura de capital (MACHADO et al., 2016), empreendedorismo (OLUGBOLA, 2017), sistemas de controle gerencial (DAVILA; FOSTER; JIA, 2015; LOPES; BEUREN, 2018), modelos de gestão colaborativa (MAFFIA et al., 2018). De modo específico, relacionado à sustentabilidade em startups, pode-se mencionar as pesquisas desenvolvidas por Galpin e Hebard (2015), Winkler, Ozturk e Brown (2016), Franceschelli, Santoro e Candelo (2018).

Face ao apresentado, a lacuna de pesquisa percebida perpassa as abordagens das publicações sobre a temática de sustentabilidade no ambiente de startups. Assim, neste estudo objetiva-se analisar as abordagens dos artigos publicados em periódicos internacionais sobre sustentabilidade no ambiente de startups. Assim, foi realizada uma revisão sistemática de artigos, cuja técnica atua como um mecanismo na busca por identificar, avaliar e sintetizar as evidências relevantes disponíveis (GALVÃO; PEREIRA, 2014). Além de identificar as publicações sobre a temática nos últimos 20 anos (1999-2018), os periódicos e a autoria, verificaram-se aspectos metodológicos e os objetivos declarados dos artigos.

A prática de ações relacionadas à sustentabilidade corporativa é geralmente abordada pela literatura como algo enfatizado por grandes corporações, porém, mesmo que mais recentemente e com menor intensidade a área de startups sustentáveis e empreendedorismo tem recebido contribuições relevantes (BOCKEN, 2015). De acordo com Schaltegger e Wagner (2011), o tamanho da organização 
não é, de forma isolada, um critério que define o empreendedorismo sustentável. Isto denota a relevância da investigação da sustentabilidade em diferentes organizações, independente do porte, dentre as quais estão as startups.

A revisão sistemática de artigos teórico-empíricos que abordam sobre a sustentabilidade no contexto organizacional de startups pode contribuir para a consolidação da literatura desta temática. Nesta revisão é possível identificar lacunas para o desenvolvimento de outras pesquisas. Além disso, pode-se verificar tendências em relação ao que tem sido abordado como eixo central das pesquisas, identificar as principais formas de coleta e análise dos dados, de modo que possam ser reproduzidas em outros contextos organizacionais ou utiliza-las em pesquisas que versem sobre diferentes temáticas no ambiente de startups.

\section{Plataforma teórica}

Nesta seção faz-se uma incursão teórica na sustentabilidade organizacional e na sustentabilidade do ambiente de startups, com vistas na fundamentação das análises dos resultados desta pesquisa.

\subsection{Sustentabilidade organizacional}

A sustentabilidade organizacional advém da necessidade de reversão dos impactos negativos oriundos das atividades desenvolvidas pelas organizações (SRIDHAR, 2010). A sustentabilidade pode ser considerada a partir da ênfase de três diferentes perspectivas, que juntas formam o Triple Bottom Line (TBL) (LANGE, 2017). Segundo Slaper e Hall (2011), também é comumente chamado de três "Ps": pessoas, planeta e lucros. O TBL foi inicialmente uma abordagem discutida por Elkington (1994), com o intuito de expandir a responsabilidade organizacional, tradicionalmente focada em questões econômicas, para incluir as dimensões sociais e ambientais (WILSON, 2015).

A perspectiva da responsabilidade econômica está voltada à questão da criação de valor para o cliente o ao desempenho financeiro da organização (BANSAL, 2005). Para mensurar as questões econômicas relacionadas à sustentabilidade são consideradas variáveis que lidam com a linha de fundo e o fluxo de dinheiro, dentre as 
quais estão tamanho do estabelecimento, crescimento de emprego, distribuição de emprego por setor, receita por setor, dentre outras (SLAPER; HALL, 2011).

A visão social da sustentabilidade foca nas questões relacionadas à equidade social, relações com a comunidade, ética, saúde e segurança no ambiente de trabalho, dentre outras (SIMPSON; RADFORD, 2014; CALDAS; TAMBOSI FILHO; VIEIRA, 2014). A mensuração dos aspectos sociais da sustentabilidade compreende elementos relacionados à educação, equidade e acesso aos recursos sociais, saúde e bem-estar, qualidade de vida e capital social, o que leva a consideração de variáveis como taxa de emprego, taxa de participação feminina na força de trabalho e expectativa de vida (SLAPER; HALL, 2011).

A perspectiva ambiental inclui ações voltadas à ecologia, preocupações com a proteção dos recursos naturais, visando a redução, por exemplo, de recursos não renováveis (BANSAL, 2005). As variáveis consideradas para a análise ambiental devem compreender medidas de recursos naturais e refletir potenciais influências na sua viabilidade, e compreende, por exemplo, consumo de energia elétrica, gestão de resíduos sólidos, consumo de combustíveis fósseis, mudança no uso da terra/cobertura do solo (SLAPER; HALL, 2011).

As perspectivas da sustentabilidade podem apresentar intersecções. De acordo com Elkington (2001), quando se considera aspectos econômicos e ambientais, pode-se obter ecoeficiência, o que permite a redução de custos dos impactos ambientais. Outro exemplo apontado pelo autor é a relação entre elementos econômicos e sociais, o que culmina na geração de ética empresarial, que denota a forma como a organização se relaciona com a sociedade. Pode-se ainda observar a interação entre aspectos de natureza social e ambiental, o que acaba por desenvolver justiça ambiental, elemento responsável pela equidade, desencadeando senso de necessidade de se fazer investimentos que beneficiem indivíduos e comunidade (ELKINGTON, 2001).

De modo geral, os aspectos econômicos da sustentabilidade são compreendidos, o que não acontece com as questões relativas aos elementos da sustentabilidade ambiental e social (GIMENEZ; SIERRA; RODON, 2012). Isso talvez possa estar relacionado ao 
fato das empresas acreditarem que os aspectos ambientais e sociais dificultam a obtenção de retornos financeiros. Todavia, Gimenez, Sierra e Rodon (2012) argumentam que ganhos financeiros positivos podem ser obtidos no processo, de modo que as organizações não precisam apenas se engajar em um comportamento social e ambiental responsável.

Govindan, Khodaverdi e Jafarian (2013) advertem que a sustentabilidade se tornou relevante no contexto das pesquisas e práticas organizacionais em decorrência do esgotamento dos recursos naturais, além de preocupações com a riqueza e responsabilidade social corporativa. As organizações são avaliadas por parte das organizações interessadas em realizar investimentos, assim como pelos entes públicos, por suas práticas voltadas à sustentabilidade (OZANNE et al., 2016).

Nos debates que acontecem no ambiente organizacional, o termo sustentabilidade está cada vez mais em evidência. A preocupação com os impactos causados ao ambiente e à sociedade acabou por desenvolver nas organizações o senso de responsabilidade além do valor decorrente de suas atividades (HUBBARD, 2009). A promoção de práticas sustentáveis de um negócio começa pela incorporação da sustentabilidade na definição das estratégias organizacionais, com adoção clara de que a sustentabilidade é parte da missão, valores e metas estabelecidas (GALPIN; HEBARD, 2015).

\subsection{Sustentabilidade no ambiente de startups}

As startups são modelos organizacionais tidos como de natureza temporária, que buscam modelos de negócios de escala, repetitivos e lucrativos, diferentes daqueles encontrados em outras empresas que operam em mercados maduros (LAUŽIKAS et al., 2015). Além disso, acabam por propiciar alternativas para as organizações na busca por modelos de negócios direcionados a aspectos de sustentabilidade (SAHUT; PERIS-ORTIZ, 2014) e inovação (WEIBLEN; CHESBROUGH, 2015).

Para atender diferentes demandas externas e o que é requerido pelas partes interessadas nas negociações, as startups precisam adotar práticas de negócios voltadas à sustentabilidade (SCHICK; MARXEN; FREIMANN, 2002). Todavia, os autores apontam que é 
importante considerar que o processo de implementação de práticas sustentáveis no contexto das organizações é geralmente uma iniciativa com desafios gigantescos e prolongados.

A orientação ambiental de startups tem recebido atenção crescente pelos pesquisadores, investidores e formuladores de políticas (BERGSET; FICHTER, 2015; DOGANOVA; KARNØE, 2015; SCHIEDERIG; TIETZE; HERSTATT, 2012). Quando as startups são orientadas para questões de natureza ambiental, acabam por contribuir consideravelmente para o desenvolvimento sustentável, uma vez que podem trabalhar com processos de inovação ambiental ou mesmo pressionar as empresas de maior porte já estabelecidas (HART; CHRISTENSEN, 2002; HOCKERTS; WUESTENHAGEN, 2010).

Diferindo-se de startups convencionais, as startups sustentáveis adotam abordagem baseada em valor e na intenção de iniciar mudanças sociais e ambientais na sociedade onde se inserem (HOCKERTS; WÜSTENHAGEN, 2010). Destacam-se por promover o desenvolvimento de negócios que buscam o sucesso para além da base de clientes verdes, baseando-se em novas tecnologias e em plataformas diferenciadas de financiamento (BOCKEN, 2015).

Nesta perspectiva, torna-se oportuno concentrar-se em startups como fonte de sustentabilidade e inovação de modelos de negócios, uma vez que tais organizações adotam modelos que incentivam o compartilhamento de espaços, o que é considerado uma proposta mais sustentável em comparação ao que é adotado por concorrentes (BOCKEN, 2015). Isso sugere que o ambiente de startups favorece o desenvolvimento de modelos de negócios com vistas na sustentabilidade e na inovação.

As startups que desejam agregar valor no mercado em que atuam e se diferenciar das demais, precisam incorporar o TBL em suas inovações (WEISSBROD; BOCKEN, 2017). Essas organizações encontram oportunidades em modelos de inovação de negócios equilibrados pelo modelo do TBL, ou seja, compreendem aspectos que envolvem questões econômicas, sociais e ambientais (CAIADO et al., 2018).

Depreende-se que os modelos de negócios sustentáveis compreendem elementos ambientais e sociais, além dos tradicionais 
interesses financeiros. Argumenta-se que a ênfase conjunta nas três diferentes perspectivas do TBL é relevante no processo de direcionamento e implementação de inovações relacionadas à sustentabilidade organizacional (BOCKEN et al., 2014). Esse processo parece ser inerente às startups com orientação estratégica voltada para questões econômicas, sociais e ambientais.

\section{Metodologia}

Este estudo foi desenvolvido a partir de uma revisão sistemática de pesquisas teórico-empíricas sobre sustentabilidade no ambiente de startups. A revisão sistemática compreende a análise de estudos relevantes sobre um assunto/tema, na busca por avaliar e sintetizar informações sobre os materiais selecionados por meio de métodos previamente determinados (GROSS; GAO; HUANG, 2013). A revisão sistemática é uma forma de análise que auxilia na replicação de pesquisas, ao oferecer a possibilidade de confirmação de decisões, procedimentos e conclusões apresentados pelas pesquisas revisadas (BOWEN et al., 2010).

$\mathrm{Na}$ execução da pesquisa observaram-se os procedimentos propostos por Tranfield, Denyer e Smart (2003), que compreendem planejamento, pesquisa, triagem e extração, síntese e relatório. No processo de planejamento, foi observado o objetivo da pesquisa para delimitar os elementos de busca na base de dados, como palavras-chave e período de busca. Quanto a seleção da base de dados, passo inicial para a pesquisa, optou-se pelo banco de dados da Scopus, por sua relevância acadêmica, com elevado padrão editorial e científico, o que proporciona maior confiabilidade aos materiais selecionados.

Na base de dados da Scopus procedeu-se à busca por trabalhos que apresentam os termos "startup" or "startups" or "start-up" or "start-ups" and "sustainability" no título, resumo ou palavras-chave. Para delimitar, optou-se ainda pelo filtro relativo ao período de publicação dos artigos, que compreendeu os últimos 20 anos quando da realização da pesquisa dos materiais, ou seja, do período de 1999 a 2018, além de se direcionar a busca apenas por trabalhos classificados como "artigos". Assim, foram obtidos 256 artigos.

Outro filtro realizado na seleção dos artigos foi o campo de pesquisa sobre gestão, o que levou à seleção de estudos classificados nas 
áreas temáticas "Negócios, Gestão e Contabilidade", e resultou na amostra de 77 artigos. Dos artigos selecionados nessa etapa, pode-se perceber que a temática sustentabilidade em startups começou a se destacar a partir do ano de 2010, com a publicação de 10 artigos. Destacam-se ainda as publicações realizadas nos anos de 2016, 2017 e 2018, com, respectivamente, 13, 10 e 12 artigos.

Após a seleção descrita foi desenvolvida a análise geral dos artigos com foco no resumo, introdução e aspectos metodológicos. Os dados coletados foram organizados e tabulados em uma planilha, com destaque para as informações relativas ao título, autores, ano de publicação, canal de publicação, objetivo, segmento de atuação das startups, país(es) onde operam e aspectos metodológicos da pesquisa.

Ao realizar a revisão analítica dos artigos selecionados, excluíram-se 41 deles por não apresentarem informações suficientemente claras, pela falta de acesso ao artigo completo ou por não estarem relacionados ao objetivo deste estudo. Foram considerados desconexos do objetivo do estudo os artigos voltados à revisão de literatura, modelos conceituais ou de negócios, simulação, percepções de estudantes e indivíduos não envolvidos com startups, ou não demonstraram foco na análise dessas empresas startups. Assim, a amostra final da pesquisa compreendeu um total de 36 artigos.

As informações relativas aos autores, ano das publicações, periódicos e aspectos metodológicos foram tabuladas em uma planilha geral. As questões metodológicas foram analisadas em conformidade com a classificação proposta por Martins e Theóphilo (2009), como segue: abordagem quanto às estratégias de pesquisa (bibliográfica, documental, experimental, quase-experimental, levantamento, estudo de caso, pesquisa-ação, etnográfica, grouded theory e discurso do sujeito coletivo); e técnicas de coleta de informações, dados e evidências (observação, observação participante, pesquisa documental, entrevista, laddering, painel, focus group, questionário, escalas sociais e de atitudes, história oral e história de vida, análise de conteúdo e análise de discurso).

Para as etapas finais propostas por Tranfield, Denyer e Smart (2003), foram analisados os objetivos dos artigos selecionadas e apontados os principais aspectos levantados nos artigos, com des- 
taque para os resultados encontrados pelas pesquisas mais recentes, com vistas à compreender as propostas desenvolvidas.

\section{DESCRIÇÃo E ANÁlise doS RESUltados}

Nesta seção apresenta-se a análise descritiva dos 36 artigos que compuseram a amostra final da pesquisa, compreendendo o ano das publicações, periódicos e autoria dos artigos. Na sequência discorreu-se sobre aspectos metodológicos das pesquisas. Por fim, fez-se uma explanação sobre os objetivos declarados nos artigos que compõem a amostra da pesquisa.

\subsection{Análise descritiva dos artigos selecionados}

A pesquisa compreendeu a busca por artigos teórico-empíricos sobre sustentabilidade em startups, publicados em periódicos internacionais no período de 1999 e 2018. Na Figura 1 apresenta-se a distribuição dos 36 artigos no período analisado.

Figura 1. Distribuição das publicações no período de 1999 a 2018

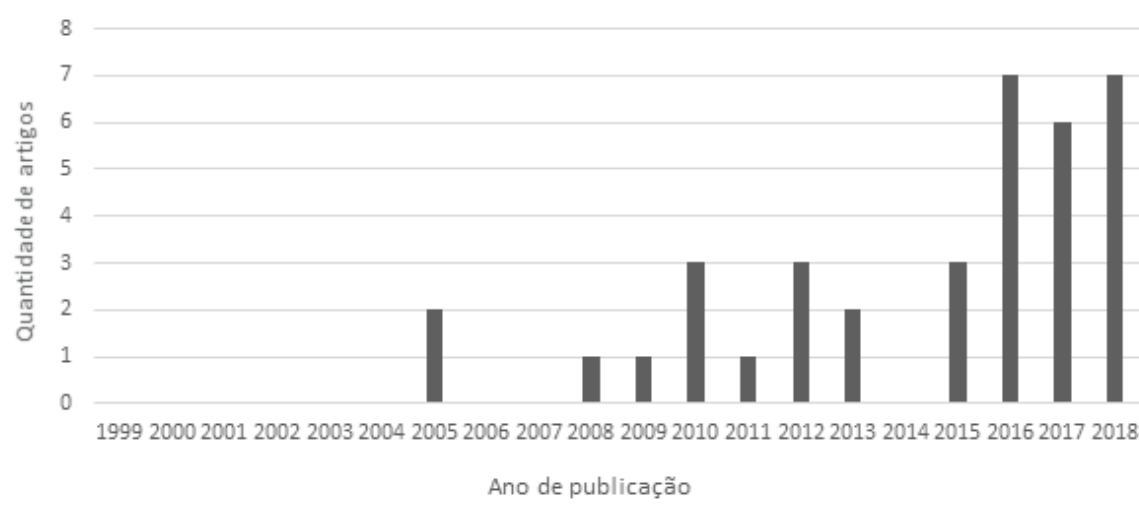

Fonte: Dados da pesquisa.

Em análise da amostra final de trabalhos selecionados, não foram observados artigos publicados nos anos 1999, 2000, 2001, 2002, 2003, 2004, 2006, 2007 e 2014. Destaca-se que os anos com maior quantidade de publicações foram 2016, 2017 e 2018, respectivamente, 
com sete, seis e sete publicações. Em seguida constam os anos de 2010, 2012 e 2015 com três publicações cada, depois os anos de 2005 e 2013 com duas publicações. Os demais anos analisados apresentaram apenas uma publicação cada.

Os 36 artigos que compõem a amostra da pesquisa foram publicados em 30 periódicos distintos da base de dados da Scopus. Na Figura 2 exibe-se a distribuição pelos periódicos dos artigos publicados sobre o tema investigado.

Figura 2. Distribuição pelos periódicos dos artigos publicados

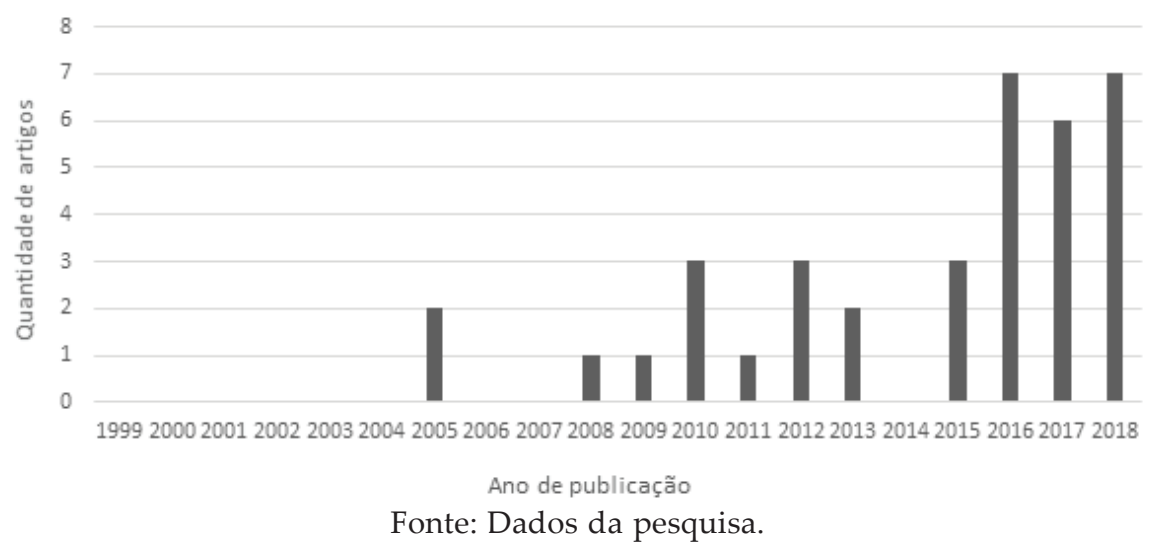

Os periódicos que mais apresentaram publicações sobre a temática sustentabilidade no ambiente de startups no período analisado foram: Journal of Cleaner Production com seis publicações, sendo uma em 2016, duas em 2017 e três em 2018, e o Industry and Higher Education com duas publicações, sendo uma no ano de 2005 e outra em 2016. Os demais periódicos analisados apresentaram apenas uma publicação cada.

Ao identificar os autores que publicaram sobre a temática sustentabilidade no ambiente de startups, não se observou autores mais prolíferos em comparação aos demais. Destaque apenas para Monica Diochon, Teresa V. Menzies e Yvon Gasse que juntos publicaram dois artigos, sendo um em 2005 e outro em 2008, e para Natasha Bank que publicou dois artigos em coautoria com outros autores, sendo um em 2016 e outro em 2017. 


\subsection{Aspectos metodológicos dos artigos pesquisados}

Em relação aos aspectos metodológicos, o primeiro elemento a ser considerado remete à abordagem da pesquisa quanto aos objetivos, isto é, todos os artigos analisados são de natureza teórico-empírica. Quanto ao problema apresentado, 21 artigos apresentaram características que levaram à classificação como pesquisa qualitativa e 13 pesquisas adotaram a abordagem quantitativa. Além disso, duas pesquisas adotaram tanto aspectos de natureza qualitativa como quantitativa.

Considerando a classificação apresentada por Martins e Theóphilo (2009), na Tabela 2 apresenta-se a quantificação dos artigos analisados no que tange aos aspetos da abordagem da estratégia de pesquisa e as técnicas de coleta de informações, dados e evidencias.

\section{Tabela 1. Classificação quanto à estratégia de pesquisa e técnicas de coleta}

\begin{tabular}{|c|c|c|c|c|c|}
\hline \multirow{2}{*}{$\begin{array}{l}\text { Abordagem quanto às } \\
\text { estratégias de pesquisa }\end{array}$} & \multicolumn{2}{|c|}{ Quantidade } & \multirow{2}{*}{$\begin{array}{c}\text { Técnicas de coleta de } \\
\text { informações, dados e } \\
\text { evidências }\end{array}$} & \multicolumn{2}{|c|}{ Quantidade } \\
\hline & Absoluta & Relativa & & Absoluta & Relativa \\
\hline Pesquisa bibliográfica & - & - & Observação & 4 & $7 \%$ \\
\hline Pesquisa documental & 1 & $3 \%$ & Observação participante & - & - \\
\hline Pesquisa experimental & - & - & Pesquisa documental & 16 & $30 \%$ \\
\hline Pesquisa quase experimental & - & - & Entrevista & 18 & $33 \%$ \\
\hline Levantamento & 15 & $39 \%$ & Laddering & - & - \\
\hline Estudo de caso (ou casos) & 18 & $47 \%$ & Painel & - & - \\
\hline Pesquisa-ação & 2 & $5 \%$ & Focus group & - & - \\
\hline Pesquisa etnográfica & - & - & Questionário & 13 & $24 \%$ \\
\hline Grounded Theory & 1 & $3 \%$ & Escalas sociais e de atitudes & - & - \\
\hline Discurso do Sujeito Coletivo & - & - & História oral e história de vida & - & - \\
\hline \multirow[t]{3}{*}{ Não informado/não está claro } & 1 & $3 \%$ & Análise de conteúdo & - & - \\
\hline & & & Análise do discurso & - & - \\
\hline & & & Não informado/não está claro & 3 & $6 \%$ \\
\hline Total & 38 & $100 \%$ & Total & 54 & $100 \%$ \\
\hline
\end{tabular}

Nota: A amostra final é de 36 artigos, mas as quantidades absolutas de trabalhos são distintas desse valor e entre si, uma vez que alguns artigos adotaram mais de uma classificação ou utilizaram mais de uma forma de coleta.

Fonte: Dados da pesquisa. 
Observa-se na Tabela 1, que os artigos analisados adotaram predominantemente as técnicas de estudo de caso (47\%) ou levantamento (39\%). Em relação às técnicas para a coleta de informações, dados e evidências, destaca-se a adoção de entrevistas (33\%), pesquisa documental (30\%) e questionários (24\%). A maioria dos estudos de caso analisados adotou como técnica de coleta as entrevistas, questionários e observação, o que permite a triangulação, um dos requisitos desta técnica.

As análises das informações, dados e evidências realizadas nos artigos selecionados, consistiram de análise descritiva pautada na transcrição de textos provenientes de entrevistas e/ou de documentos, com o emprego de estatística descritiva ou técnicas estatísticas como ANOVA, regressão, análise de cluster, análise fatorial, modelagem de equações estruturais e coeficiente de contingência de Cramer V.

Quanto aos respondentes das pesquisas que fizeram uso de questionário ou entrevistas, destaca-se que de modo geral foram requisitadas informações junto aos gestores ou proprietários/empreendedores/ fundadores. No entanto, há pesquisas que contaram com entrevistas ou questionários aplicados aos clientes, especialistas da indústria da moda, gestores de incubadoras, membros diversos da cadeia de suprimentos e outros agentes relacionados (não especificados).

No que concerne aos países onde as startups investigadas pelos estudos analisados operam suas atividades, seis artigos consideraram startups que operam em diferentes países, cinco observaram empresas dos Estados Unidos, quatro analisaram empresas da Alemanha, três investigaram empresas localizadas no Canadá ou Itália ou Reino Unido. Alguns países foram considerados em apenas uma pesquisa cada. Uma pesquisa foi desenvolvida considerando empresas localizadas em países como China, Dinamarca, Equador, Nigéria, Noruega e República Checa. Em um artigo não se identificou o país sede da startup analisada.

Em relação ao segmento de atuação das startups ou incubadoras analisadas pelos artigos, pode-se verificar que 18 artigos pesquisaram empresas de diferentes segmentos, sendo que o seu foco era obtenção de percepções dos empreendedores ou gestores. Para as pesquisas que optaram pelo estudo de um tipo exclusivo de empresas, não houve segmentos analisados em mais de uma pesquisa, 
sendo consideradas empresas que atuam no ramo de alimentos, atividades rurais, moda, reciclagem e reaproveitamento, tecnologia e turismo.

\subsection{Abordagens da sustentabilidade nos artigos}

A temática sustentabilidade pode ser compreendida sob três diferentes abordagens, econômica, social e ambiental, que juntas compõem o Triple Bottom Line (TBL). Nesta perspectiva, o objetivo declarado nos artigos analisados é apresentado na Tabela 2.

\section{Tabela 2. Objetivo dos artigos analisados}

\begin{tabular}{l|l|lc}
\hline Ano & Autor(es) & Objetivo & $\begin{array}{l}\text { Aborda- } \\
\text { gem TBL }\end{array}$ \\
\hline 2005 & $\begin{array}{l}\text { Pam Voisey, Lynne } \\
\text { Gornall, Paul Jones } \\
\text { e Brychan Thomas }\end{array}$ & $\begin{array}{l}\text { Explorar e compreender o funcionamento das } \\
\text { incubadoras de empresas e o ambiente onde atuam. }\end{array}$ & Econômica \\
\hline 2005 & $\begin{array}{l}\text { Monica Diochon, } \\
\text { Teresa V. Menzies e } \\
\text { Yvon Gasse }\end{array}$ & $\begin{array}{l}\text { Analisar características dos empreendedores associados } \\
\text { a empreendimentos startups. }\end{array}$ & Econômica \\
\hline 2008 & $\begin{array}{l}\text { Monica Diochon, } \\
\text { Teresa V. Menzies e } \\
\text { Yvon Gasse }\end{array}$ & $\begin{array}{l}\text { Explorar a natureza e o impacto do capital humano da } \\
\text { gestação na partida bem-sucedida de empreendedores } \\
\text { de startups canadenses. }\end{array}$ & Econômica \\
\hline 2009 & $\begin{array}{l}\text { G. Page West III e } \\
\text { Terry W. Noel }\end{array}$ & $\begin{array}{l}\text { Analisar o impacto no desempenho de novos } \\
\text { empreendimentos como resultado de diferentes fontes } \\
\text { de conhecimento, e como essas relações são exploradas } \\
\text { em novas empresas de base tecnológica. }\end{array}$ & Econômica \\
\hline 2010 & $\begin{array}{l}\text { Michael Lewrick e } \\
\text { Robert Raeside }\end{array}$ & $\begin{array}{l}\text { Explorar a dinâmica por trás das inovaçóes, quais são } \\
\text { as capacidades necessárias em cada etapa do ciclo } \\
\text { de vida das empresas e os desafios para inovar na } \\
\text { transformação. }\end{array}$ & Econômica \\
\hline \multirow{2}{*}{2010} & Jan Claussen e \\
Eleanor O'Higgins & $\begin{array}{l}\text { Apresentar um modelo de escolha do consumidor de } \\
\text { companhias aéreas para analisar proposições de valor } \\
\text { - os benefícios oferecidos aos passageiros em relação às } \\
\text { tarifas pagas. }\end{array}$ & Econômica \\
\hline
\end{tabular}




\begin{tabular}{|c|c|c|c|}
\hline Ano & Autor(es) & Objetivo & $\begin{array}{l}\text { Aborda- } \\
\text { gem TBL }\end{array}$ \\
\hline 2011 & $\begin{array}{l}\text { Abigail R. Clarke- } \\
\text { Sather, Margot J. } \\
\text { Hutchins, Qiong } \\
\text { Zhang, John K. } \\
\text { Gershenson e John } \\
\text { W. Sutherland }\end{array}$ & $\begin{array}{l}\text { Considerar duas abordagens diferentes de avaliação de } \\
\text { sustentabilidade realizadas em um estudo de caso de } \\
\text { uma única empresa, uma startup PME. }\end{array}$ & Econômica \\
\hline 2012 & $\begin{array}{l}\text { Marco Caliendo, } \\
\text { Jens Hogenacker, } \\
\text { Steffen Künn e } \\
\text { FrankWießner }\end{array}$ & $\begin{array}{l}\text { Fornecer evidências sobre a estrutura do participante } \\
\text { do novo programa de financiamento em comparação } \\
\text { com os antigos programas. }\end{array}$ & Econômica \\
\hline 2012 & Anat BarNir & $\begin{array}{l}\text { Entender melhor os fatores associados à decisão dos } \\
\text { empreendedores de incorporar tecnologias inovadoras } \\
\text { em novos empreendimentos. }\end{array}$ & Econômica \\
\hline 2012 & $\begin{array}{l}\text { Lucia Naldi e } \\
\text { Robert G. Picard }\end{array}$ & $\begin{array}{l}\text { Explorar como os fatores presentes em startups de } \\
\text { notícias on-line influenciam seu desenvolvimento e } \\
\text { sustentabilidade. }\end{array}$ & Econômica \\
\hline 2013 & $\begin{array}{l}\text { Fernando Lourenço, } \\
\text { Oswald Jones e } \\
\text { Dilani Jayawarna }\end{array}$ & $\begin{array}{l}\text { Adaptar a Teoria do Comportamento Planejado } \\
\text { para examinar atitudes em relação a uma forma } \\
\text { empreendedora de educação da sustentabilidade. }\end{array}$ & Econômica \\
\hline 2013 & $\begin{array}{l}\text { Tommy D. } \\
\text { Andersson, Don } \\
\text { Getz e Reidar J. } \\
\text { Mykletun }\end{array}$ & $\begin{array}{l}\text { Abordar a sustentabilidade das populações de } \\
\text { festivais a partir da perspectiva da Teoria da Ecologia } \\
\text { Organizacional e, em particular, da dependência de } \\
\text { idade e densidade. }\end{array}$ & $\begin{array}{l}\text { Econômica/ } \\
\text { Social }\end{array}$ \\
\hline 2015 & $\begin{array}{l}\text { Mohammed Bala } \\
\text { Banki e Hairul } \\
\text { Nizam Ismail }\end{array}$ & $\begin{array}{l}\text { Contribuir para o entendimento das microempresas de } \\
\text { turismo de propriedade familiar na Montanha Obudu, } \\
\text { um destino turístico na Nigéria. }\end{array}$ & $\begin{array}{l}\text { Econômica/ } \\
\text { Social }\end{array}$ \\
\hline 2015 & $\begin{array}{l}\text { Rowena Ortiz- } \\
\text { Walters, Monica C. } \\
\text { Gavino e Denise } \\
\text { Williams }\end{array}$ & $\begin{array}{l}\text { Explorar as redes sociais dos proprietários de empresas } \\
\text { latinas e as implicações para o crescimento de risco } \\
\text { (tamanho dos funcionários) e a sustentabilidade (anos } \\
\text { no negócio) }\end{array}$ & $\begin{array}{l}\text { Econômica/ } \\
\text { Social }\end{array}$ \\
\hline 2015 & $\begin{array}{l}\text { Liliana Doganova e } \\
\text { Peter Karnøe }\end{array}$ & $\begin{array}{l}\text { Investigar a construção de mercados para tecnologias } \\
\text { limpas, examinando os mecanismos pelos quais as } \\
\text { novas tecnologias conseguem (ou não) se transformar } \\
\text { em bens que são ambiental e economicamente valiosos. }\end{array}$ & $\begin{array}{l}\text { Econômica/ } \\
\text { Ambiental }\end{array}$ \\
\hline 2016 & $\begin{array}{l}\text { Caterina De Lucia, } \\
\text { Pasquale Balena, } \\
\text { Maria Rosaria } \\
\text { Stufano Melone e } \\
\text { Dino Borri }\end{array}$ & $\begin{array}{l}\text { Acrescentar novos insights ao debate atual sobre } \\
\text { ingredientes ativos e investigar relações entre } \\
\text { criatividade e sustentabilidade a partir de perspectivas } \\
\text { do lado da oferta e da demanda. }\end{array}$ & Econômica \\
\hline 2016 & $\begin{array}{l}\text { Natasha Bank e } \\
\text { Wisdom Kanda }\end{array}$ & $\begin{array}{l}\text { Analisar os processos de recrutamento e suporte em } \\
\text { incubadoras com perfil de sustentabilidade. }\end{array}$ & Econômica \\
\hline
\end{tabular}




\begin{tabular}{|c|c|c|c|}
\hline Ano & Autor(es) & Objetivo & $\begin{array}{l}\text { Aborda- } \\
\text { gem TBL }\end{array}$ \\
\hline 2016 & $\begin{array}{l}\text { Louise Canning e } \\
\text { Isabelle Szmigin }\end{array}$ & $\begin{array}{l}\text { Examinar o processo de rede e o papel da competência } \\
\text { da rede em trazer ao mercado alternativas radicais } \\
\text { à tecnologia de cremação e para as quais o benefício } \\
\text { potencial para a sociedade requer o envolvimento de } \\
\text { um amplo conjunto de atores. }\end{array}$ & Econômica \\
\hline 2016 & $\begin{array}{l}\text { Kalinga Jagoda, } \\
\text { Xiaohua Lin, } \\
\text { Victoria Calvert e } \\
\text { Shaw Tao }\end{array}$ & $\begin{array}{l}\text { Examinar a utilização de agências de apoio a } \\
\text { empreendimentos e organizações comunitárias por } \\
\text { proprietários de PMEs rurais e a satisfação desses. }\end{array}$ & Econômica \\
\hline 2016 & Jarmila Šebestová & $\begin{array}{l}\text { Comparar e contrastar a relação entre a automotivação } \\
\text { em startup e avaliar o ambiente de negócios na região } \\
\text { da Morávia-Silésia. }\end{array}$ & Econômica \\
\hline 2016 & $\begin{array}{l}\text { Eduardo Barrientos } \\
\text { e Anne H. Reilly }\end{array}$ & $\begin{array}{l}\text { Descrever o início da startup, sua missão e plano de } \\
\text { negócios, e as suas realizações até à data, juntamente } \\
\text { com recomendações para outras startups da linha de } \\
\text { fundo da empresa social. }\end{array}$ & Econômica \\
\hline 2016 & $\begin{array}{l}\text { George } \\
\text { Malindretos, } \\
\text { Konstantinos } \\
\text { Tsiboukas e Sofia } \\
\text { Argyropoulou- } \\
\text { Konstantaki }\end{array}$ & $\begin{array}{l}\text { Analisar a exploração das oportunidades derivadas } \\
\text { da produção de vinho e gestão de resíduos, dentro } \\
\text { do ambiente totalmente novo de negócios, econômico, } \\
\text { social e físico. }\end{array}$ & Econômica \\
\hline 2017 & $\begin{array}{l}\text { Bruna Villa } \\
\text { Todeschini, } \\
\text { Marcelo Nogueira } \\
\text { Cortimiglia, } \\
\text { Daniela Callegaro- } \\
\text { de-Menezes e } \\
\text { Antonio Ghezzi }\end{array}$ & $\begin{array}{l}\text { Investigar modelos de negócios inovadores na indústria } \\
\text { da moda que tenham sustentabilidade como sua } \\
\text { característica definidora, especialmente em termos de } \\
\text { proposição de valor. }\end{array}$ & Econômica \\
\hline 2017 & $\begin{array}{l}\text { Boyd Cohenme } \\
\text { Pablo Muñoz }\end{array}$ & $\begin{array}{l}\text { Explorar como uma gama de empresas abordou a } \\
\text { entrada no mercado consumidor consciente. }\end{array}$ & Econômica \\
\hline 2017 & $\begin{array}{l}\text { Natasha Bank, } \\
\text { Klaus Fichter e } \\
\text { Magnus Klofsten }\end{array}$ & $\begin{array}{l}\text { Investigar como as incubadoras sustentáveis garantem a } \\
\text { entrada de inquilinos, como organizam suas atividades } \\
\text { e se o ambiente da incubadora afeta o recrutamento de } \\
\text { inquilinos. }\end{array}$ & Econômica \\
\hline 2017 & $\begin{array}{l}\text { Deborah E. de } \\
\text { Lange }\end{array}$ & $\begin{array}{l}\text { Examinar se a sustentabilidade é recompensada pelos } \\
\text { investidores de startups. }\end{array}$ & Econômica \\
\hline 2017 & $\begin{array}{l}\text { Katherina Kuschel, } \\
\text { María-Teresa } \\
\text { Lepeley e Silvia } \\
\text { Fernanda Espinosa } \\
\text { Sebastián Gutiérrez }\end{array}$ & $\begin{array}{l}\text { Analisar os desafios que as fundadoras de startups } \\
\text { enfrentam para garantir o financiamento no setor de } \\
\text { tecnologia. }\end{array}$ & Econômica \\
\hline
\end{tabular}




\begin{tabular}{|c|c|c|c|}
\hline Ano & Autor(es) & Objetivo & $\begin{array}{l}\text { Aborda- } \\
\text { gem TBL }\end{array}$ \\
\hline 2017 & $\begin{array}{l}\text { Yuchen Gao e } \\
\text { Yimei } \mathrm{Hu}\end{array}$ & $\begin{array}{l}\text { Explorar os principais fatores e formas específicas para } \\
\text { a atualização das incubadoras híbridas no contexto da } \\
\text { China. }\end{array}$ & Econômica \\
\hline 2018 & $\begin{array}{l}\text { Nancy M. P. } \\
\text { Bocken, Ruth } \\
\text { Mugge, Colin A. } \\
\text { Bom e Hidde-Jan } \\
\text { Lemstra }\end{array}$ & $\begin{array}{l}\text { Analisar o impacto ambiental positivo em termos de } \\
\text { melhoria dos padrões de consumo observado em um } \\
\text { modelo de negócio de pagamento por uso. }\end{array}$ & Ambiental \\
\hline 2018 & $\begin{array}{l}\text { Maria Vittoria } \\
\text { Franceschelli e } \\
\text { Gabriele Santoro } \\
\text { and Elena Candelo }\end{array}$ & $\begin{array}{l}\text { Investigar e apresentar a maneira pela qual uma startup } \\
\text { de alimentos pode desenvolver inovações no modelo de } \\
\text { negócios, levando em conta a importância das questões } \\
\text { sociais e ambientais. }\end{array}$ & $\begin{array}{l}\text { Social/ } \\
\text { Ambiental }\end{array}$ \\
\hline 2018 & $\begin{array}{l}\text { Corinna } \\
\text { Dickenbrok e Luis } \\
\text { F. Martinez }\end{array}$ & $\begin{array}{l}\text { Analisar a comunicação de startups de eco-moda em } \\
\text { diferentes culturas e regiões geográficas. }\end{array}$ & Social \\
\hline 2018 & $\begin{array}{l}\text { Petra Dickel, Jacob } \\
\text { Horisch e Thomas } \\
\text { Ritter }\end{array}$ & $\begin{array}{l}\text { Analisar o impacto da orientação ambiental externa e } \\
\text { interna sobre as características da rede de startups. }\end{array}$ & Econômica \\
\hline 2018 & $\begin{array}{l}\text { Thomas B. Long, } \\
\text { Arnold Looijen e } \\
\text { Vincent Blok }\end{array}$ & $\begin{array}{l}\text { Explorar e identificar fatores críticos de sucesso e } \\
\text { barreiras para a transição de modelos de negócios } \\
\text { tradicionais para modelos de negócios voltados à } \\
\text { sustentabilidade. }\end{array}$ & Econômica \\
\hline 2018 & $\begin{array}{l}\text { Federico Cosenz e } \\
\text { Guido Noto }\end{array}$ & $\begin{array}{l}\text { Discutir sobre uma ferramenta de design de estratégia } \\
\text { baseada na combinação entre esquemas convencionais } \\
\text { de representação de modelo de negócio e modelagem } \\
\text { de System Dynamics (SD). }\end{array}$ & Econômica \\
\hline 2018 & $\begin{array}{l}\text { Yadira Mariela } \\
\text { Arteaga Estrella, } \\
\text { Rosa Aurora } \\
\text { Espinoza } \\
\text { Toalombo, Xiomara } \\
\text { Leticia Zuñiga } \\
\text { Santillán, Eduardo } \\
\text { Javier Espinoza } \\
\text { Solís, Félix Enrique } \\
\text { Villegas Yagual } \\
\text { e Hugo Federico } \\
\text { Campos Rocafuerte }\end{array}$ & $\begin{array}{l}\text { Relacionar empreendimentos turísticos com } \\
\text { desenvolvimento sustentável do turismo no Cantão de } \\
\text { Milagro (Equador). }\end{array}$ & Econômica \\
\hline
\end{tabular}

Fonte: Dados da pesquisa. 
De acordo com a Tabela 2, os estudos investigaram diferentes aspectos de sustentabilidade em startups. A amplitude do escopo encontrado nas pesquisas pode decorrer da dificuldade de delimitar o significado do termo sustentabilidade, que vai desde assegurar a continuidade do empreendimento até a sustentabilidade do ambiente e outras conexões que o temo permite inferir. A análise das publicações na base de dados da Scopus indica ainda que no período de 2016 a 2018 foram publicadas mais pesquisas que buscaram desvendar as preocupações das startups quanto a sustentabilidade social e ambiental.

A sustentabilidade é destacada como um elemento positivo no contexto organizacional, mas o processo de adoção de modelos sustentáveis de negócios é desafiador. Todavia, as startups parecem um campo propício para testar estes novos modelos, o que é sinalizado com mais pesquisas recentes sobre o tema. Na Figura 3 destacam-se as abordagens dos artigos à luz do TBL, segregadas em econômicas, sociais e ambientais.

\section{Figura 3. Abordagens do Triple Bottom Line nos artigos publicados}

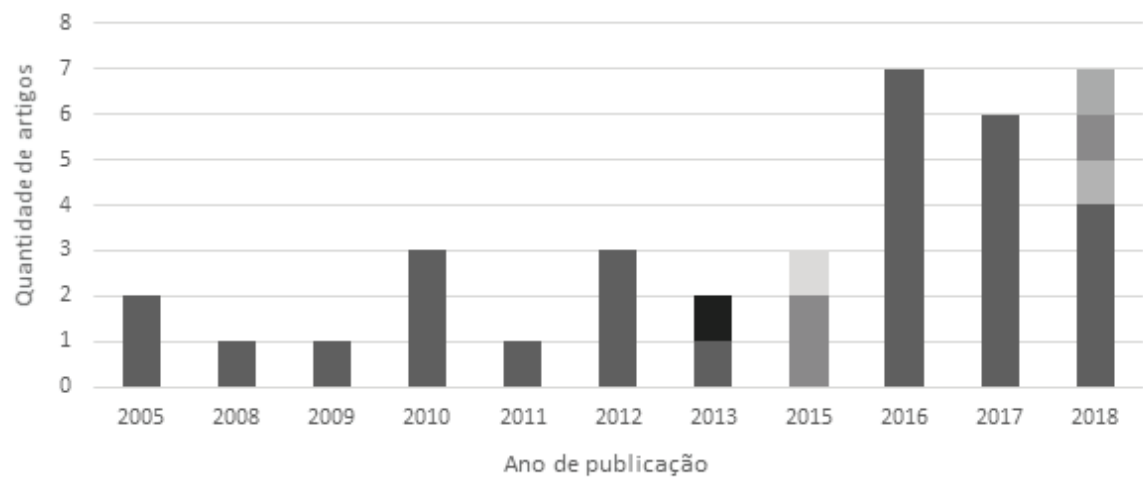

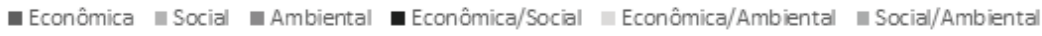
Fonte: Dados da pesquisa.

Ao analisar os artigos que compõem a amostra final da pesquisa, observa-se que, de modo geral, os trabalhos foram desenvolvidos com foco em questões da sustentabilidade econômica (31 artigos), 
estando em segundo plano os artigos que apresentam abordagens mais voltadas aos aspectos ambientais (cinco artigos), seguidos da abordagem social (três artigos). Destaca-se que alguns artigos apresentaram características que permitiram a classificação em mais de uma abordagem do TBL, conforme apontado por Elkington (2001). $\mathrm{O}$ incremento recente das publicações sobre as preocupações de startups em desenvolver negócios com foco na sustentabilidade do planeta e saúde das pessoas pode estar associado com o número de novos empreendimentos dessa natureza emergentes no mundo todo.

$\mathrm{Na}$ análise dos artigos são encontradas pesquisas voltadas à verificação de elementos de empreendedorismo e características empreendedoras dos indivíduos que atuam nestas organizações. Nessa linha, tem-se trabalhos como os de Diochon, Menzies e Gasse (2005), Rodgers (2010) e Kuschel et al. (2017), que abordaram sobre características de empreendedores, empreendedorismo sustentável e desafios de empreendedores de empresas startups para garantir financiamento.

Diochon et al. (2005) constataram que alguns aspectos de natureza pessoal acabam por diferenciar os empreendedores que se desligam no processo inicial do negócio, daqueles que dão continuidade, com destaque para questões relativas ao estilo de resolução de problemas e à orientação para objetivos. Rodgers (2010) verificou que os negócios estão se preocupando não somente com questões monetárias, sendo essas fortemente condicionadas pela natureza eco-consciente do negócio na busca por metas sustentáveis. Kuschel et al. (2017) encontraram fatores relacionados às necessidades de capital, redes e características individuais como sendo determinantes para a obtenção de recursos, e que a busca por financiamento pode ser vista como fonte de empregabilidade e sustentabilidade econômica.

Outro aspecto observado é que as empresas startups apresentam um forte direcionamento para questões relacionadas à inovação. Assim, a temática inovação tende a estar presente nas abordagens sobre sustentabilidade, o que é corroborado nas pesquisas de Lewrick e Raeside (2010), BarNir (2012) e Todeschini et al. (2017).

Lewrick e Raeside (2010) investigaram a dinâmica por trás das inovações, observando as capacidades necessárias e os desafios para 
inovar na transformação. O estudo evidenciou a importância da orientação para o mercado, conhecimento, capacidades de gestão, redes sociais para a inovação, sucesso e sustentabilidade, quando se considera a transformação de startups da fase inicial para a fase de maior maturidade. BarNir (2012) buscou compreender os fatores associados à decisão dos empreendedores de incorporar tecnologias inovadoras em novos empreendimentos. A pesquisa pode identificar a diferenciação entre as percepções de homens e mulheres e contribuir ao evidenciar a importância da compreensão de fatores que motivam e encorajam os empreendedores a utilizar tecnologias inovadoras para profissionais, educadores e formuladores de políticas. Todeschini et al. (2017) investigaram modelos de negócios inovadores na indústria da moda que têm a sustentabilidade como característica definidora. Observaram que a orientação do modelo de negócios para a sustentabilidade no campo moda tende a ser marcadamente diferente para os operadores tradicionais e iniciantes. Além disso, apesar da utilidade da tecnologia, esse não é um requisito difícil para modelos de negócios de moda inovadores de sucesso.

Uma lente diferenciada é observada na pesquisa de Long, Looijen e Blok (2018), que buscaram explorar e identificar fatores críticos de sucesso e barreiras para a transição de modelos de negócios tradicionais para modelos de negócios voltados à sustentabilidade. Como resultados da pesquisa destacaram a questão de que organizações que almejam desenvolver o modelo de negócios voltado à sustentabilidade, devem tornar a sustentabilidade o princípio-chave sobre o qual a empresa é fundada. Além disso, faz-se necessário o desenvolvimento e melhorias contínuas, com suporte de membros da rede da qual a empresa faz parte, tais como fornecedores, clientes e governo.

\section{Considerações Finais}

Este estudo analisou as abordagens das pesquisas publicadas em periódicos sobre a temática sustentabilidade no ambiente de startups, com destaque para o período com maior número de publicações, os periódicos que se sobressaíram, a autoria dos artigos, aspectos metodológicos das pesquisas e o objetivo declarado em cada artigo. Os resultados da pesquisa indicaram que há uma tendência de 
crescimento de publicações sobre a temática nos vinte anos analisados, com maior concentração das publicações nos últimos dez anos considerados. Essa observação coaduna com o exposto por Hockerts e Wüstenhagen (2010), que apontaram o crescimento no corpo da literatura que versa sobre sustentabilidade e startups, com destaque para a adoção do conceito de empreendedorismo social, que começou a emergir no final da década de 1990, tornando-se foco de recentes pesquisas acadêmicas.

Em relação aos aspectos metodológicos que permeiam as pesquisas dos artigos analisados, verificaram destaques na abordagem quanto às estratégias de pesquisa, com prevalência do uso corrente de estudos de casos e levantamentos, bem como das técnicas de coleta de informações, dados e evidências, em que se sobressaiu a utilização de questionários, análise de documentos e realização de entrevistas. Todavia, não se conseguiu detectar um segmento que está em voga quando o assunto é sustentabilidade em startups.

Os objetivos declarados nos artigos investigados evidenciam que não há um eixo quanto ao que é pretendido nos artigos analisados. Desse modo, não se consegue fazer inferências gerais quanto às tendências das pesquisas que abordam sobre aspectos da sustentabilidade em startups. Nessa linha, não foi possível perceber harmonização entre o que foi abordado pelos artigos como proposta central sobre a sustentabilidade em startups. Ressalta-se, no entanto, que várias pesquisas versaram sobre questões que abordam de alguma forma o empreendedorismo e características empreendedoras, além da preocupação com inovação sustentável.

A análise da temática sustentabilidade no contexto de startups sob a lente econômica, social e ambiental, que compõem o Triple Bottom Line (TBL), pelos objetivos declarados nos artigos pesquisados, revela que há prevalência da abordagem de aspectos econômicos. Isso sugere que há uma lacuna de pesquisa na abordagem da sustentabilidade no contexto de startups, o envolvimento das demais lentes, visto que organizações que buscam inovar de forma sustentável devem focar não apenas na eficiência econômica, mas visar também maior responsabilidade social e ambiental (BARBIERI et al., 2010).

À guisa de implicações, destaca-se a importância de se tratar sobre aspectos sustentáveis no ambiente de startups, já que acabam 
por fornecer diferenciais competitivos (BOCKEN et al., 2014). Além disso, a incorporação da sustentabilidade em startups acaba por ser algo interessante, já que novas empresas apresentam poucos ou nenhum processo ou procedimento organizacional estabelecido (GALPIN; HEBARD, 2015), o que facilita a formulação de estratégias sustentáveis.

Dentre as limitações deste estudo aponta-se a natureza amostral e temporal, uma vez que se delimitou a pesquisa à análise apenas das publicações disponibilizadas na base de dados da Scopus e ao período de apenas vinte anos (1999-2018). A definição dos termos de busca também representa uma limitação, visto que restringe o escopo das publicações possíveis. É importante considerar ainda questões relativas à interpretação ou de acesso a determinadas informações nos artigos analisados. Nessa linha, novos estudos podem ser desenvolvidos considerando outras bases de dados, expansão do período de tempo compreendido e diferentes combinações de termos de busca de artigos. Recomenda-se inclusive a análise dos artigos encontrados por meio de outras técnicas de revisão.

\section{REFERÊNCIAS}

BANSAL, P. Evolving sustainably: A longitudinal study of corporate sustainable development. Strategic Management Journal, v. 26, n. 3, p. 197-218, 2005.

BARBIERI, J. C.; VASCONCELOS, I. F. G.; ANDREASSI, T.; VASCONCELOS, F. C. Inovação e sustentabilidade: Novos modelos e proposições. RAE. Revista de Administração de Empresas, v. 50, n. 2, p. 146-154, 2010.

BARNIR, A. Starting technologically innovative ventures: Reasons, human capital, and gender. Management Decision, v. 50, n. 3, p. 399-419, 2012.

BERGSET, L.; FICHTER, K. Green start-ups-a new typology for sustainable entrepreneurship and innovation research. Journal of Innovation Management, v. 3, n. 3, p. 118-144, 2015.

BOCKEN, N. M. P. Sustainable venture capital-catalyst for sustainable start-up success? Journal of Cleaner Production, v. 108, p. 647-658, 2015.

BOCKEN, N. M. P.; RANA, P.; SHORT, S. W. Value mapping for sustainable business thinking. Journal of Industrial and Production Engineering, v. 32, n. 1, p. 67-81, 2015.

BOCKEN, N. M. P.; SHORT, S. W.; RANA, P.; EVANS, S. A literature and practice review to develop sustainable business model archetypes. Journal of Cleaner Production, v. 65, p. 42-56, 2014. 
BOWEN, F.; NEWENHAM-KAHINDI, A.; HERREMANS, I. When suits meet roots: The antecedents and consequences of community engagement strategy. Journal of Business Ethics, v. 95, n. 2, p. 297-318, 2010.

CAIADO, R. G. G.; QUELHAS, O. L. G.; NASCIMENTO, D. L. M.; COUTINHO, G. C.; MEIRIÑO, M. J. Synergic framework between Lean philosophy and Triple Bottom Line in the entrepreneurial environment. Journal of Lean Systems, v. 3, n. 2, p. 76-89, 2018.

CALDAS, C. B.; TAMBOSI FILHO, E.; VIEIRA, A. M. Governança Corporativa e Sustentabilidade: uma relação necessária. Revista UNIABEU, v. 7, p. 353-369, 2014.

DAVILA, A.; FOSTER, G.; JIA, N. The valuation of management control systems in start-up companies: International field-based evidence. European Accounting Review, v. 24, n. 2, p. 207-239, 2015.

DIOCHON, M.; MENZIES, T. V.; GASSE, Y. Canadian nascent entrepreneurs' start-up efforts: Outcomes and individual influences on sustainability. Journal of Small Business \& Entrepreneurship, v. 18, n. 1, p. 53-74, 2005.

DOGANOVA, L.; KARNØE, P. Building markets for clean technologies: Controversies, environmental concerns and economic worth. Industrial Marketing Management, v. 44, p. 22-31, 2015.

DULLIUS, A. C.; SCHAEFFER, P. R. As capacidades de inovação em startups: Contribuições para uma trajetória de crescimento. Revista Alcance, v. 23, n. 1, p. 34-50, 2016.

ELKINGTON, J. Canibais com garfo e faca. São Paulo: Makron Books, 2001.

FRANCESCHELLI, M. V.; SANTORO, G.; CANDELO, E. Business model innovation for sustainability: A food start-up case study. British Food Journal, v. 120, n. 10, p. 2483-2494, 2018.

Galpin, T.; Hebard, J. Sustainability in start-up ventures: What founders say versus what they do. World Journal of Entrepreneurship, Management and Sustainable Development, v. 11, n. 4, p. 246-255, 2015.

GALVÃO, T. F.; PEREIRA, M. G. Revisões sistemáticas da literatura: Passos para sua elaboração. Epidemiologia e Serviços de Saúde, v. 23, 183-184, 2014.

GIMENEZ, C.; SIERRA, V.; RODON, J. Sustainable operations: Their impact on the triple bottom line. International Journal of Production Economics, v. 140, n. 1, p. 149-159, 2012.

GOVINDAN, K.; KHODAVERDI, R.; JAFARIAN, A. A fuzzy multi criteria approach for measuring sustainability performance of a supplier based on triple bottom line approach. Journal of Cleaner Production, v. 47, n. 345-354, 2013.

GROSS, M. J.; GAO, H.; HUANG, S. S. China hotel research: A systematic review of the English language academic literature. Tourism Management Perspectives, v. 6, p. 68-78, 2013.

HALL, J. K.; DANEKE, G. A.; LENOX, M. J. Sustainable development and entrepreneurship: Past contributions and future directions. Journal of Business Venturing, v. 25, n. 5, p. 439448, 2010.

HART, S. L.; CHRISTENSEN, C. M. The great leap: Driving innovation from the base of the pyramid. MIT Sloan Management Review, v. 44, n. 1, p. 51-56, 2002. 
HOCKERTS, K.; WÜSTENHAGEN, R. Greening Goliaths versus emerging Davids:Theorizing about the role of incumbents and new entrants in sustainable entrepreneurship. Journal of Business Venturing, v. 25, n. 5, p. 481-492, 2010.

HUBBARD, G. Measuring organizational performance: Beyond the triple bottom line. Business Strategy and the Environment, v. 18, n. 3, p. 177-191, 2009.

KUSCHEL, K.; LEPELEY, M. T.; ESPINOSA, F.; GUTIÉRREZ, S. Funding challenges of Latin American women start-up founders in the technology industry. Cross Cultural \& Strategic Management, v. 24, n. 2, p. 310-331, 2017.

LANGE, D. E. Start-up sustainability: An insurmountable cost or a life-giving investment? Journal of Cleaner Production, v. 156, p. 838-854, 2017.

LAUŽIKAS, M.; TINDALE, H.; BILOTA, A.; BIELOUSOVAITĖ, D. Contributions of sustainable start-up ecosystem to dynamics of start-up companies: The case of Lithuania. Entrepreneurship and Sustainability Issues, v. 3, p. 8-24, 2015.

LEWRICK, M.; RAESIDE, R. Transformation and change process in innovation models: Start-up and mature companies. International Journal of Business Innovation and Research, v. 4, n. 6, p. 515-534, 2010.

LONG, T. B.; LOOIJEN, A.; BLOK, V. Critical success factors for the transition to business models for sustainability in the food and beverage industry in the Netherlands. Journal of Cleaner Production, v. 175, p. 82-95, 2018.

LOPES, I. F.; BEUREN, I. M. Reflexos da cultura local nos sistemas de controle de incubadoras de empresas. Revista de Contabilidade e Organizações, v. 12, p. 1-14, 2018.

MACHADO, E.; SELIG, P. M.; FOLLMANN, N.; CASAROTTO FILHO, N. Análise da influência do capital estrutural no sucesso de startups incubadas: Uma pesquisa com 21 empreendedores. International Journal of Innovation, v. 4, n. 1, p. 46-57, 2016.

MAFFIA, L. F. C. M.; COUTO, M. H. G.; SANTOS, R. F.; OLIVA, F. L.; HILDEBRAND, C. C.; CORREA, H. L. Premissas e benefícios do modelo de gestão colaborativo em startups. Revista Brasileira de Gestão e Inovação, v. 6, n. 1, p. 71-94, 2018.

MARTINS, G. A.; THEÓPHILO, C. R. Metodologia da investigação científica para Ciências Sociais Aplicadas. 2. ed. São Paulo: Atlas, 2009.

OLUGBOLA, S. A. Exploring entrepreneurial readiness of youth and startup success components: Entrepreneurship training as a moderator. Journal of Innovation \& Knowledge, v. 2, n. 3, p. 155-171, 2017.

OZANNE, L. K.; PHIPPS, M.; WEAVER, T.; CARRINGTON, M.; LUCHS, M.; CATLIN, J.; GRUPTA, N. S.; SCOTT, K.; WILLIAMS, J. Managing the tensions at the intersection of the triple bottom line: A paradox theory approach to sustainability management. Journal of Public Policy \& Marketing, v. 35, n. 2, p. 249-261, 2016.

PACHECO, D. F.; DEAN, T. J.; PAYNE, D. S. Escaping the green prison: Entrepreneurship and the creation of opportunities for sustainable development. Journal of Business Venturing, v. 25, n. 5, p. 464-480, 2010. 
RODGERS, C. Sustainable entrepreneurship in SMEs: A case study analysis. Corporate Social Responsibility and Environmental Management, v. 17, n. 3, p. 125-132, 2010.

SAHUT, J. M.; PERIS-ORTIZ, M. Small business, innovation, and entrepreneurship. Small Business Economics, v. 42, n. 4, p. 663-668, 2014.

SCHALTEGGER, S.; WAGNER, M. Empreendedorismo sustentável e inovação em sustentabilidade: Categorias e interações. Estratégia de Negócios e Meio Ambiente, v. 20, n. 4, p. 222-237, 2011.

SCHICK, H.; MARXEN, S.; FREIMANN, J. Sustainability issues for start-up entrepreneurs. Greener Management International, v. 38, p. 59-70, 2002.

SCHIEDERIG, T.; TIETZE, F.; HERSTATT, C. Green innovation in technology and innovation management; An exploratory literature review. R\&D Management, v. 42, n. 2, p. 180-192, 2012.

SHORT, S. W.; BOCKEN, N. M.; BARLOW, C. Y.; CHERTOW, M. R. From refining sugar to growing tomatoes: Industrial ecology and business model evolution. Journal of Industrial Ecology, v. 18, n. 5, p. 603-618, 2014.

SIMPSON, B. J. K.; K. RADFORD, S. Situational variables and sustainability in multiattribute decision-making. European Journal of Marketing, v. 48, n. 5/6, p. 1046-1069, 2014.

SLAPER, T. F.; HALL, T. J. The triple bottom line: What is it and how does it work. Indiana Business Review, v. 86, n. 1, p. 4-8, 2011.

SRIDHAR, K. A multi-dimensional criticism of the Triple Bottom Line reporting approach. International Journal of Business Governance and Ethics, v. 6, n. 1, p. 49-67, 2010.

TODESCHINI, B. V.; CORTIMIGLIA, M. N.; CALLEGARO-DE-MENEZES, D.; GHEZZI, A. Innovative and sustainable business models in the fashion industry: Entrepreneurial drivers, opportunities, and challenges. Business Horizons, v. 60, n. 6, p. 759-770, 2017.

TRANFIELD, D.; DENYER, D.; SMART, P. Towards a methodology for developing evidence-informed management knowledge by means of systematic review. British Journal of Management, v. 14, n. 3, p. 207-222, 2003.

WEIBLEN, T.; CHESBROUGH, H. W. Engajar-se com startups para aprimorar a inovação corporativa. California Management Review, v. 57, n. 2, p. 66-90, 2015.

WEISSBROD, I.; BOCKEN, N. M. Developing sustainable business experimentation capability: A case study. Journal of Cleaner Production, v. 142, p. 2663-2676, 2017.

WILSON, J. P. The triple bottom line: Undertaking an economic, social, and environmental retail sustainability strategy. International Journal of Retail \& Distribution Management, v. 43, n. 4/5, p. 432-447, 2015.

WINKLER, T. J.; OZTURK, P.; BROWN, C. V. Sustainability strategies for regional health information organization startups. Health Policy and Technology, v. 5, n. 4, p. 341-349, 2016.

Recebido em: 23-8-2019

Aprovado em: 13-11-2020

Avaliado pelo sistema double blind review.

Disponível em http://mjs.metodista.br/index.php/roc 